\title{
An overview of Synthetic Jet under Different Clamping and Amplitude Modulation Techniques
}

\author{
Itimad D J Azzawi \\ Department of Mechanical Engineering, \\ College of Engineering, University of Diyala, Baquba, Diyala, Iraq. \\ Itimaddawood_eng@uodiyala.edu.iq \\ ASME Membership (\#100767130)
}

Artur J. Jaworski

University of Huddersfield / Huddersfield / HD1 3DH / UK

School of Computing and Engineering

A.Jaworski@hud.ac.uk

Xiaoan Mao

University of Leeds / Leeds / LS2 9JT/ UK

School of Chemical and Process Engineering

X.Mao@leeds.ac.uk

\begin{abstract}
There is generally limited guidance available on the optimum clamping method for the diaphragms used in the SJAs. This paper describes the effects of clamping methods (O-rings, neoprene rubber washers and metal-to-metal clamping) on the actuator diaphragm displacement using Polytec Scan Vibrometer (PSV). Once the clamping type was implemented, an optimization study to examine the effect of geometrical parameters for three designs of synthetic jet actuators in quiescent conditions - in particular number of orifices per cavity, the space between them and their effects on the jet velocity were investigated. It has also been shown that use the Helmholtz resonance of the cavity and amplitude modulation of the excitation signal, the actuator can exhibit a more significant "blowing" velocity at a reduced power input.
\end{abstract}

\section{Introduction and Previous Literature Related}

Synthetic jet actuators (SJAs) have gained considerable attention in recent decades. Their potential areas of application are wide-ranging from aerospace flow control, including flow separation [1-4], jet/thrust vectoring [5, 6], flow mixing in chemical engineering $[7,8]$ and heat transfer enhancement $[9,10]$. The excitation types of SJA may vary to include electrodynamic excitation (e.g. loudspeaker type), mechanical (e.g. reciprocating piston) or piezo ceramic [11]. The latter are particularly convenient due to their lightweight, performance reliability and repeatability and ease of electrical excitation. 
Figure 1 shows a typical SJA comprising of three basic components; namely, a piezo-ceramic diaphragm, an orifice plate and a cavity in between. The oscillation of piezo-ceramic diaphragm located at the bottom of the cavity determines the periodic changes in the cavity's volume. As a result, the external fluid is entrained into the cavity during the downward movement (so called "suction" phase) and ejected to the surroundings during an upward movement (so-called "blowing" or "ejection" phase). The key part of any SJA is the oscillatory diaphragm. Piezoelectric discs have been the most widely used as active diaphragms in SJAs due to their low weight and low power consumption. In the last 15 years, different piezoelectric composites have been developed for the purpose of producing higher displacement as well as improved longevity. The piezoelectric composites used in actuators include various types, most popular being unimorphs [12], bimorphs [13], thunders [14], Radial Field Diaphragms (RFDs) [15] or Lipca diaphragms [16].

Radial Field Diaphragms (RFD) have been also developed by NASA as distinctly different design from traditional piezoceramic actuators. In either form the main feature is the use of the $\mathrm{d} 33$ coupling mode: i.e. the application of electric field in the direction 3 correspond to piezo-electric effect also in direction 3. Moreover, the coupling coefficient $\mathrm{d} 33$ tends to be 3-4 times higher than $\mathrm{d} 31$. Therefore, the diaphragm's perpendicular displacement is higher [17]. More information about these two modes can be seen in [18-21]. Since synthetic jet actuators have zero net mass injection over their system boundary, synthetic jets are also known as zero-net-mass-flux (ZNMF) devices. Hence, they constitute a useful and cost-effective option for a wide range of applications as mentioned earlier. To enhance the flow ejected from a typical SJA, the synthetic jet actuator parameters design (e.g. oscillatory types, operational and geometrical parameters) that affect the formation and evaluation of the vortex rings in quiescent flow should be investigated. Knowing how such parameters are coupled in the production of a given jet and understanding their contribution towards achieving a given objective of such application is important when designing effective actuators. The synthetic jet formation and velocity can be explained through a number of operational parameters, such as excitation frequencies and amplitude [22], velocity ratio [23], oscillatory types [11, 15, 16], waveform types [17] and amplitude modulation technique [22].

Clearly, the diaphragm is only one part of a synthetic jet actuator required for its operation. It is placed at the actuator cavity as shown in Figure 1 and appropriately excited to enable the generation of vortex rings. The actuator as whole can then be characterised by different parameters as mentioned above. These can be classified in detail into two groups that can define the vortex ring, jet velocity and jet strength which will be taken into a consideration in the current study. The first group is known as the geometrical parameters including cavity height $(H)$, cavity diameter $\left(D_{c}\right)$, orifice diameter $\left(D_{0}\right)$, and orifice depth (L). The second group is known as operating parameters, which include excitation frequency, the orifices distribution and 
amplitude modulation. The geometrical and operating parameters both have a direct impact on the peak velocity and formation of the synthetic jets that they create.

In actual applications of SJAs there is an important consideration of the acoustic resonance taking place inside the cavity (so called Helmholtz frequency) which will be discussed later. Regarding the geometrical parameters, Jain et al [24] found that the jet velocity was almost constant at different cavity heights. Yang [12] on the other hand, observed that when the cavity height was reduced, the jet velocity was increased [25]. Higher orifice thickness (L) would result in higher circulation, however lower jet velocity [26].

It has also been shown that the cavity and orifice shapes have a significant effect on the jet strength and jet velocity [27, 28]. Perhaps the most widely used types of active diaphragms in SJAs design are unimorphs and bimorphs due to their low cost (below a \$1 mark), low weight, low power consumption and their ability to generate a considerable peak jet velocity [14, 16 and 23]. These actuators are designed so that the excitation parameters such as voltage and mechanical resonance (in unclamped conditions) are usually in the range from 0 to \pm 100 Volts and 1 to $3 \mathrm{kHz}$, respectively. However, the methods used in clamping of these diaphragms have a significant influence on the final performance of the diaphragm, especially in terms of the mechanical resonance frequency and their deformation. Therefore, the clamping mechanism used becomes critical to the characterisation of the SJA and special attention to its design need to be considered. Moreover, in most applications these diaphragms are excited at frequencies lower than their resonance frequency to increase the fatigue life of the bonded ceramic [22].

There is generally very little guidance available from the existing literature on the optimum clamping method for the diaphragms used in the SJAs. Jabbal et al [29] suggested using a nitrile O-ring can provide a good air sealing while, another researcher claimed that clamping SJA between two O-rings damped out its displacement capabilities [14]. Therefore, to introduce better understanding of how different clamping techniques can affect the SJA, a Polytec Scaning Vibrometer (PSV) was used to investigate the effect of the clamping method on diaphragm displacement and hence in the jet peak velocity. Then an optimisation of single jet actuator was conducted using different number of orifices and space between them. Moreover, studies and literature reviews on the effect of the Helmholtz frequency and amplitude modulation methodology on the synthetic jet formation and velocity profile are limited, compared to un-modulated operation research. Therefore, the operational parameters, such as the mechanical driving frequency, Helmholtz frequency and amplitude modulation, on jet peak velocity are presented and discussed. The knowledge gained from the above experiments allowed the application of the developed SJA to be used in a flow control application. 


\section{Experimental Apparatus and Measurement Techniques}

Three different setups are described in this section. The first setup is used to investigate the effect of the diaphragm clamping method on its displacement using a Polytec Scanning Vibrometer (PSV). The second setup is introduced to examine the effects of geometrical parameters on the jet formation and peak jet velocity in quiescent conditions (i.e. without inserting the devices into the crossflow).

\subsection{Quiescent condition setup}

\subsubsection{Diaphragm clamping}

The active element used in the construction of the synthetic jet actuators is the piezo-ceramic diaphragm Model: APC FT27T-3.9A1. It is incorporated in the actuator assemblies by a common housing and three different clamping mechanisms. The housing was machined out of aluminium. The geometrical parameters of the baseline actuator unit are indicated in Table 1. Three clamping types are shown in Figure 2a: a pair of O-rings, a one-sided neoprene rubber washer (cf. Figure 2b), and metal-to-metal (cf. Figure 2c). In all three cases, a metal clamp was used to push the diaphragm and/or the seal material against a shoulder provided inside the cavity by tightening four screws. An assembled actuator unit was held in a standard laboratory stand with a clamp. As the excitation frequency is applied, the deflection of the piezo-ceramic element is determined using the laser scanning vibrometer.

The baseline dimensions of the actuator explained in Table 1 were designed in such away it can be varied by adding appropriate attachments to aluminium plates when is needed, which provided a simple way to vary the orifice number and space between them. The baseline cavity height studied were $3 \mathrm{~mm}$, while the orifice diameters were $1.2 \mathrm{~mm}$. This way of manufacturing the actuator unit allowed for a convenient way to study the effect of geometrical parameters on jet formation and jet velocity. The scanning laser vibrometer is a PC controlled instrument that provides velocity measurement at multiple points. The laser moves automatically to each point and scans of the whole area under investigation at predefined grid-points. Once this scan is completed, an animation of the deflection shape can be visualised. The Polytec Scanning Vibrometer, PSV400 from Polytec consists of a controller (OFV-5000), a junction box and a PC system equipped with a 4 channel $5 \mathrm{MHz}$ sampling card from National Instruments. The velocity and displacement decoders are the main parts of the controller. More information available from [30].

Figure 3 shows how the displacement of an isolated diaphragm (i.e. without any cavity attached) was studied using the Polytec Scanning Vibrometer (PSV). For each clamping method, the diaphragm was excited with a sine sweep over the frequency range $10 \mathrm{~Hz}$ to $3 \mathrm{kHz}$ (in $2 \mathrm{~Hz}$ increments) while the measuring spatial and temporal distribution of the actuator vibration. 
The clamping methods applied to the piezo-ceramic diaphragm disc reduced the maximum allowable excitation voltage from $60 \mathrm{~V}$ with a free boundary condition to about $50 \mathrm{~V}(35 \mathrm{Vrms})$ as bench top tests had demonstrated that the fatigue life of the bonded ceramic on the disks was significantly impaired for voltages $(\mathrm{V}>35 \mathrm{Vrms})$ and frequencies nearer the mechanical resonance frequency. The photograph of the experimental layout and its block diagram, including the instrumentation used, are shown in Figure 4. The velocity was measured using hot-wire anemometry using IFA 300 constant temperature anemometer (CTA). The hot-wire sensor was positioned above the actuator orifice through a $5 \mathrm{~mm}$ probe support to measure the jet velocity. A two-axis manually controlled traversing mechanism with a $250 \mathrm{~mm} \times 250 \mathrm{~mm}$ travel distance was used to position the probe (c.f. Figure 4). The in-plane time-averaged velocity was measured in both x-plane and in the z plane.

The driving circuitry that supplies the excitation signal to the piezo-ceramic diaphragm used consists of two components. The first one is the function generator, which allows the waveform type and excitation frequency to be altered as required over the range $10 \mathrm{~Hz}$ to $3 \mathrm{kHz}$. The output from the function generator was fed to a PDm-200 power amplifier (piezo-driver), which can provide a voltage amplitude ranging from 0 to 100 volts. The output from the amplifier is used to excite the piezo-ceramic diaphragm.

In the case of synthetic jet applications whereby high circulation is required with little input energy, reducing the orifice diameter whilst at the same time increasing the number of orifices quite advantageous [31, 32]. The vortex ring circulation is a significant parameter which measures the vortex ring strength. A strong vortex ring produces high jet axial momentum. Therefore, the use of multiple-orifice actuators could facilitate the possibility of using this type of actuator geometry for applications that require a significant introduction of systematic circulation into the flow. The question now is whether increasing the number of orifices of a particular actuator can improve the actuator efficiency or otherwise.

To address this question, hot-wire tests were performed on single, double and multiple orifice synthetic jet actuators in order to investigate the advantages of multiple orifice actuators over the one orifice actuator to achieve higher circulation. It is vital that vortex rings are formed and developed individually without interacting with each other to avoid any circulation cancellation [31]. Therefore, two dominant parameters featured in the present study, the spacing between the orifices to explore their effect on the vortex rings produced in one, two and three orifice actuators. The optimum orifice space was experimentally investigated, as shown in Figure 5 to compare between two orifices spaced, at $5 \mathrm{~mm}$ and $10 \mathrm{~mm}$ respectively.

\section{Results and Discussion}


The experimental results are divided into two main sections. Section 3.1 deals with the effect of the clamping method on the actuator performance. Section 3.2 deals with the effects of orifice geometry along the operating parameters on the actuator performance in the quiescent conditions.

\subsection{Clamping method effect}

As mentioned previously, due to the lack of guidance available from the existing literature on the optimum clamping method for the diaphragms used in the SJAs. Therefore, as the first step, it was necessary to test a number of possible clamping designs. As explained previously, three clamping types were investigated using PSV to examine which one could provide the highest jet velocity as well as ensure a strong seal and prevent any leaks which could have a detrimental effect on the actuator performance. For each clamping type, the diaphragm was excited with a sine wave of variable frequency to cover a frequency sweep between $10 \mathrm{~Hz}$ and $3 \mathrm{kHz}$ (in $2 \mathrm{~Hz}$ increments) while measuring the spatial distribution of the diaphragm velocity. At each excitation of frequency, diaphragm vibration visualization was obtained which was accompanied by the displacement data.

Figures $6 \mathrm{a}-\mathrm{b}$ show that the diaphragm had different modes of displacement at different clamping conditions. This allowed a quick and easy verification of the uniformity of the boundary condition all around the actuator circumference. It is clear that the metal to metal clamping produced the best azimuthal uniformity of boundary condition and motion with the highest velocity compared to the other two clamping methods. Clamping the diaphragm between two O-rings was the worst technique of fastening (see Figure 6a) and this was agreed with Urgina, 2010 [14] and opposed to Jabbal et al. [29], which indicated that clamping the diaphragm between two O-rings could result in improved durability by relaxing the clamping condition and thus bending stress during operation. The membrane appears to be vibrating at a combination of the first and fourth radial modes. The presence of high radial modes is unwanted, as only the first radial mode causes a radially monotonic displacement that is most effective in changing the cavity volume and thereby ejecting its fluid. In comparison, clamping the diaphragm using metal to metal and Neoprene rubber does not reveal any significant variations in the azimuthal uniformity of boundary condition and motion as shown in Figure 6b.

Figure 7 shows that the mechanical resonance frequency of the piezo-ceramic diaphragm was reduced from $2500 \mathrm{~Hz}$ without clamping (from the product data sheet) to $2200 \mathrm{~Hz}$ when clamping was applied. The latter is also useful from the point of view of selection of the operating frequency when trying to avoid mechanical resonance. Moreover, a maximum membrane displacement amplitude of $0.027 \mathrm{~mm}$ was achieved with the metal to metal clamping method. The most likely reason was that metal to metal clamping provided lower mechanical damping, while still holding the diaphragm in place. However, as can be 
inferred from Figure 7, for O-ring clamping, the displacement is decreased by $61 \%$ compared to metal to metal clamping at the same operating conditions. Furthermore, Figure 7 shows only one resonant frequency without the Helmholtz frequency of the cavity, as the cavity plate was not yet inserted. Due to its good performance, the metal-to-metal clamping method was used for the remainder of this study.

To summarise, the lack of proper clamping in general have implications on the overall SJA efficiency such that the mechanical frequency of the diaphragm is function of its geometry, mechanical properties and its clamping condition [33, 34]. Therefore, it is better to find a lower frequency to excite the SJA because most of researcher has considered either resonance frequency of the diaphragm or close to it. However, in many studies, a simple bench-top fatigue tests had shown that, for the highest frequency use (resonance frequency of the diaphragm) any high amplitude resulted in a dramatically reduced actuator lifecycle [22]. As such this was taken to be limited the frequency/amplitude required to drive the SJA. Therefore, this was pushed the researchers to find an alternative excitation frequency to drive the actuator.

\subsection{Evaluation of SJAs in quiescent conditions}

Once the optimum method of clamping has been determined, the next experimental results are divided into three main sections. Section 3.2.1 deals with the impact on actuator efficiency of geometric parameters in terms of number of orifices, distance between them, cavity height and orifice diameter, and how those parameters can be related to both mechanical and Helmholtz resonance frequencies. Section 3.2.2 enables the selection of the SJA parameters that provide the highest output based on the section 3.2.1 results. Finally, section 3.2.3 evaluates the effect of modulated and unmodulated excitation waveforms on SJA velocity at various modulated frequencies.

\subsubsection{Evaluation of the SJA geometric parameters}

Figure 8 shows a baseline SJA velocity magnitude history over two cycles of the input voltage sine wave. There are two notable velocity magnitude peaks, one large which lags the input signal maximum (+ve) and a smaller one which lags the input signal minimum (-ve). These velocity magnitude peaks are created during the duty cycle, with the larger peak being associated with the blowing cycle and the smaller peak being associated with the ingestion cycle. Figure 9 shows the z-plane velocity magnitude measured by the hot-wire probe placed at different heights above the middle orifice exit plane, along the orifice centreline, between $\mathrm{Y} / \mathrm{D}_{\mathrm{o}}=0.5$ to $\mathrm{Y} / \mathrm{D}_{\mathrm{o}}=6$. An excitation voltage of $35 \mathrm{Vrms}$ was used. Flow reversal or the suction phase is observed as a second peak at $\mathrm{Y} / \mathrm{D}_{\mathrm{o}}=0.5$, which results from the hot-wire measurements not being sensitive to the direction of the flow. This second peak is caused by the variation in the effective cross-sectional area of the synthetic jet actuator during the two halves of the cycle and this is what causes this second peak to have a lower jet velocity compared to 
the blowing phase. As the hot-wire probe was moved stream-wise further away from the orifice exit plane, to $\mathrm{Y} / \mathrm{D}_{\mathrm{o}}=4$ and 6 respectively, this second peak disappears completely. This difference increases as the probe is moved away from the orifice exit plane, until, at $\mathrm{Y} / \mathrm{D}_{\mathrm{o}}=6$, only jet-like exit velocity is measurable. This feature enables to identify relatively easily the parts of the velocity magnitude signal which related to the blowing cycle or the suction cycle.

To examine whether the vortex rings and coherent structures are produced at the actuation frequency, the power spectral density (PSD) of the velocity magnitude at two axial distances of $0.5 \mathrm{D}_{\mathrm{o}}$ and $15 \mathrm{D}_{\mathrm{o}}$ above the orifice is shown in Figure 10 . The output signal was essentially sampled at $200 \mathrm{kHz}$ which is higher than the frequency of the signal and hence the Nyquist frequency $(\mathrm{Fn}=\mathrm{Fs} / 2=100 \mathrm{kHz})$ is implemented. Moreover, based on the number of data points used in the FFT and sampling frequency, the frequency resolution was about $12.2 \mathrm{~Hz}$. Figure 10 clearly shows a harmonic series at $\mathrm{Y} / \mathrm{D}_{\mathrm{o}}=0.5 \mathrm{with}$ a $1 \mathrm{kHz}$ fundamental, which is the SJA excitation frequency. This harmonic series is essentially absent at $\mathrm{Y} / \mathrm{D}_{\mathrm{o}}=15$. This suggests the velocity fluctuations at $\mathrm{Y} / \mathrm{D}_{\mathrm{o}}=15$ are uncorrelated with the excitation frequency and/or that the synthetic jet does not reach $\mathrm{Y} / \mathrm{D}_{\mathrm{o}}=15$.

Figures 11a and b investigate the effect of orifice spacing on the measured velocity magnitude. Two orifices were tested spaced $5 \mathrm{~mm}$ and then $10 \mathrm{~mm}$ apart. The $\mathrm{x}$-plane velocity profiles measured at $\mathrm{Y} / \mathrm{D}_{\mathrm{o}}=3$ indicate that the two jets are weakly interacting when spaced $10 \mathrm{~mm}$ apart (Figure 11a) whereas the jets interact more strongly when spaced $5 \mathrm{~mm}$ apart (Figure 11b). This interaction may be detrimental to the circulation associated to the jet. This appears to be substantiated by the lower peak velocity magnitude shown in Figure $11 \mathrm{~b}$. This reduction in the peak velocity can be caused by the interaction between the edges of any vortex rings as they near each other, which may dissipate the flow energy. Therefore, for the purpose of this study, $10 \mathrm{~mm}$ was used as the minimum spacing so as to reduce the adverse jet interaction.

The effect of the number of orifices on the vortex rings circulation was studied empirically with the spacing between orifices of $10 \mathrm{~mm}$, all within the same operational parameters. The governing equation for each vortex ring as a function of the jet average velocity during the blowing phase was estimated based on the following relation [35]:

$$
\Gamma=\frac{\pi^{2}}{128} f \Delta^{2}\left(\frac{D_{c}}{D_{o}}\right)^{4}
$$

Equation 1 was used to calculate the amount of synthetic jet circulation from each orifice. To determine the total amount of SJA circulation, it is assumed that the vortex rings from multiple orifices combine together to give $\Gamma_{\text {total }}=n \Gamma_{i}, i=1, n$, and $n$ is the number of orifices. This is equivalent to invoking Kelvin's circulation theorem in the synthetic jet, away from the wall, in a barotropic fluid. By this assumption, the total circulation increases proportionally to the number of orifices. 
Therefore, having three orifices with same total area of larger single orifice increases the total vortex ring circulation by $3^{\wedge} 3$ times. As a result, a larger amount of fluid and momentum can be transferred to the cross flow. This result is encouraging. However, as this result is based on assuming an inviscid flow, this work was limited to 3 orifices per SJA, as not to generate excessive viscous losses through the neck of the orifices.

Once the number of orifices and the space between them was selected, an experiment was carried out to evaluate the effect of operating parameters frequencies and amplitude modulation on the peak velocity magnitude. The frequency response with one, two and three orifices is presented in Figure 12. In Figure 12, the peak velocity measured at $\mathrm{Y}_{\mathrm{D}}=0.5$ is shown for a constant actuation voltage of Vrms $=35$ Volts. For each orifice, two peaks of jet velocity were recorded. These two peaks defined two operation regimes of SJA, namely: Helmholtz regime and non-Helmholtz (mechanical) regime - depending on the vicinity between Helmholtz and mechanical frequencies - the former regime produced lower jet velocity compared to the non-Helmholtz regime. The Figure clearly showed that, as the number of orifices per cavity increases, Helmholtz frequency $f_{H}$ moved towards the mechanical frequency $f_{m}$ of the diaphragm. Increasing the number of orifices slightly reduces the jet velocity over each orifice.

The highest z-plane velocity magnitude peak is obtained at the diaphragm mechanical frequency of $2.2 \mathrm{kHz}$ (output velocity $15 \mathrm{~m} / \mathrm{s}$ ) and a second peak is obtained at the Helmholtz frequency of about $0.7 \mathrm{kHz}$ (output velocity $13 \mathrm{~m} / \mathrm{s}$ ). As the number of orifices is increased further from two to three, $f_{H}$ increases from 0.83 to $1 \mathrm{kHz}$ respectively. Therefore, increasing the number of orifices not only can increase the total circulation but also pushes the Helmholtz resonant frequency towards the mechanical frequency of the diaphragm. To confirm this hypothesis, the baseline dimensions of the actuator explained in Table 1 were varied by adding appropriate attachments to aluminium plates. This provided variations in the cavity height (volume) and orifice diameter by using attachments. The cavity heights studied were $3 \mathrm{~mm}$ (baseline), $5.5 \mathrm{~mm}$ and $8 \mathrm{~mm}$, while the orifice diameters were $1.2 \mathrm{~mm}$ (baseline), $2.5 \mathrm{~mm}$ and $5 \mathrm{~mm}$. Consequently, it is of interest to determine the proximity of the Helmholtz frequency to the excitation frequency of the diaphragm in different SJA geometries. For all cases, the orifice length and the cavity diameter were maintained at a constant of $1.5 \mathrm{~mm}$ and $32 \mathrm{~mm}$ respectively. The cavity height was changed from $\mathrm{H} / \mathrm{D}_{\mathrm{c}}=0.09$ to 0.17 and to 0.25 , whilst $\mathrm{L} / \mathrm{D}_{\mathrm{o}}=1.25$ to 0.6 to 0.3 . Figure 13 presents the relationship between the measured and calculated Helmholtz frequency against cavity height $\left(\mathrm{H} / \mathrm{D}_{\mathrm{c}}\right)$ for a given orifice diameter. It can be deduced from the Figure that there was a good agreement between the calculated and measured Helmholtz frequency for different cavity height. Moreover, at the orifice diameter $D_{o}=1.2 \mathrm{~mm}$, as the height of the cavity was increased from $H / D_{c}=0.09$ to 0.17 to 0.25 , the resonant frequency of the cavity decreased from $\sim 965 \mathrm{~Hz}$ to $\sim 712 \mathrm{~Hz}$ to $\sim 610 \mathrm{~Hz}$ respectively. At H/D $\mathrm{D}_{\mathrm{c}}=$ 
0.09 and $D_{o}=1.2 \mathrm{~mm}$, the excitation frequency is close to the Helmholtz frequency. As $\mathrm{H}$ increased, these two frequencies move further apart. This is likely to be detrimental to the jet velocity and may explain the trend opposite to that from Maine [16].

The velocity profiles derived from the three cavities $\left(H / \mathrm{D}_{c}=0.09,0.17\right.$ and 0.25$)$ are compared and the effects of cavity height variations at constant orifice diameters are performed. Figure 14 shows the x-plane velocity magnitude profile as a function of the cavity height, at and away from the Helmholtz frequency, $f_{H}$. Velocity magnitude profile peaks for cavity $\mathrm{H} / \mathrm{D}_{\mathrm{c}}=0.09$ and 0.17 in the case of a sine wave driving signal were reduced from $14 \mathrm{~m} / \mathrm{s}$ to $10.3 \mathrm{~m} / \mathrm{s}$ with the smaller height/volume cavity producing the higher velocity. Similarly, a cavity of $H / D_{c}=0.17$ and $H / D_{c}=0.25$ was also compared; the peak velocity magnitude was significantly reduced from $10.3 \mathrm{~m} / \mathrm{s}$ to $2 \mathrm{~m} / \mathrm{s}$. Moreover, Figure 14 clearly shows the effect of the Helmholtz frequency, $f_{H}$ on the peak velocity magnitude at a constant $\mathrm{H} / \mathrm{D}_{\mathrm{c}}$. For example, the peak velocity magnitude at $\mathrm{H} / \mathrm{D}_{\mathrm{c}}=0.17$ increased when the excitation frequency was reduced from $965 \mathrm{~Hz}$ to $712 \mathrm{~Hz}$ which was expected to be decreased, as the latter represented the Helmholtz frequency of this cavity. Similarly, at a cavity height ratio of 0.25 , the measured peak velocity magnitude increased when the excitation frequency reduced from $965 \mathrm{~Hz}$ to $f_{H}=610 \mathrm{~Hz}$, where $f_{H}$ is the Helmholtz frequency for this cavity.

\subsubsection{Selection of SJA geometrical parameters}

The results from section 3.2.1 enable the selection of the SJA parameters that provide the highest output in terms of the peak velocity magnitude for the minimum input energy. Therefore, three orifices of $1.2 \mathrm{~mm}$ orifice diameter (total area $=3.4 \mathrm{~mm}^{2}$ ) can be used. The length of the orifice and the cavity diameter are set to $1.5 \mathrm{~mm}$ and $32 \mathrm{~mm}$ respectively and a cavity length to diameter ratio $H / D_{c}=0.17$, based on the results from Figure 14 . These dimensions were also chosen as they allow SJAs to be constructed without damaging the mounting surface and provide some flexibility in the selection of the piezoelectric excitation voltage waveforms. The overall SJA geometrical dimension is shown in Figure 15.

\subsubsection{The choice of the piezoelectric excitation waveform}

Once the geometrical dimensions were chosen, it was necessary to enhance the possibility of synthetic jet structures interacting with shear layer structures of the target flow control application. The synthetic jet structures are related to the frequency and the voltages of the actuation waveform. As shown in section 3.1, the maximum orifice throughflow velocity magnitude is generated when the piezo-ceramic diaphragm is driven at the design mechanical frequency of the diaphragm. This frequency was $2200 \mathrm{~Hz}$ for the SJA of Table 1, metal-to-metal clamped. Throughout the current study the two resonant frequencies 
(Helmholtz and mechanical) are $1 \mathrm{kHz}$ and $2.2 \mathrm{kHz}$ respectively. The formal frequency (Helmholtz actuation frequencies) is used to avoid damaging the piezoceramic diaphragm if it excites at the mechanical frequency of the diaphragm.

It has been observed that synthetic jets are more effective when the forcing frequency is in the range of T-S / K-H (KelvinHelmholtz instability) frequencies of the boundary layer [36]. Therefore, two modulated frequencies $(75 \mathrm{~Hz}$ and $150 \mathrm{~Hz})$ were set to lie within the energy containing range of the spectrum of the approaching boundary layer, in fact, close to the separated boundary layer (see Figure 16) [37]. This technique has been used to evaluate the natural demodulation of the high frequency carrier (the Helmholtz frequency) with the low frequency modulating signal $(75 \mathrm{~Hz}$ and $150 \mathrm{~Hz}$ ) to enhance the flow. This presents an opportunity to perturb the flow at non-resonant frequencies and thus adds flexibility in the design of an actuator. Therefore, Figure 17 shows the time trace of the $1 \mathrm{kHz}$ carrier frequency, the amplitude modulation time trace at $75 \mathrm{~Hz}$ and the modulated waveform.

Figure 18 compares the power spectral density of the velocity magnitude measured by hot-wire at $\mathrm{Y} / \mathrm{D}_{\mathrm{o}}=0.5$ over centreline for both modulated and un-modulated input waveforms. For un-modulated case, a harmonic cascade is produced with the fundamental at the $1 \mathrm{kHz}$ peak driving frequency, which is also the Helmhotlz frequency $f_{H}$ of the cavity. When the carrier signal is modulated, two additional peaks appear at the modulating frequencies $(75 \mathrm{~Hz}$ and $150 \mathrm{~Hz})$ as shown in Figures $18 \mathrm{~b}$ and c. Modulation at $f_{m}$ also produced haystacking in the carrier frequency harmonic cascade at $n f_{H} \pm n m f_{m}$, with $\mathrm{n}$ and $\mathrm{m}$ integers. The z-plane velocity magnitude at Vrms $=35$ Volts is shown in Figures 19a to c. For the un-modulated case, the maximum velocity magnitude is $12 \mathrm{~m} / \mathrm{s}$, as shown in Figure 19a. This maximum remained essentially constant over the signal acquisition time. Moreover, when the amplitude modulation excitation is used to drive the SJA, an increase in the peak zplane velocity magnitude is achieved at both modulated frequencies. This velocity increase maximum is more likely to be caused by the constructive interference between the Helmholtz resonance and the neighbouring $f_{H} \pm m f_{m}$ frequencies. Since $f_{m} \ll f_{H}, f_{H} \pm m f_{m}$ is also close to the Helmholtz resonance frequency. The result is an "envelope" of z-plane velocity magnitude peaks at the modulation frequency. The highest peak in this envelope is higher than the un-modulated z-velocity magnitude maximum.

At the minimum modulated frequency of $75 \mathrm{~Hz}$, as shown in Figure 19b, the maximum peak velocity magnitude is $15 \mathrm{~m} / \mathrm{s}$. This is higher than the $14.2 \mathrm{~m} / \mathrm{s}$ obtained at the modulated frequency of $150 \mathrm{~Hz}$. This is likely to be due to the reduced amplification of the $f_{H} \pm m f_{m}$ frequencies as these sit further away from the Helmholtz resonance for $f_{m}=150 \mathrm{~Hz}$ than for $f_{m}=75 \mathrm{~Hz}$. Figure 20 shows that as the excitation voltage increases, both the modulated and the un-modulated peak velocity magnitude also increase. Figure supports the results shown in Figure 20 and shows that at the same excitation voltage, e.g. 30 
Vrms, the peak z-plane velocity magnitude increases from $10.5 \mathrm{~m} / \mathrm{s}$ for the un-modulated SJA to a maximum velocity of 13.5 $\mathrm{m} / \mathrm{s}$ with modulation at $f_{\mathrm{m}}=75 \mathrm{~Hz}$ and to $12.5 \mathrm{~m} / \mathrm{s}$, with modulation at $f_{\mathrm{m}}=150 \mathrm{~Hz}$. Figure 19 indicates that the largest ratio between un-modulated and modulated peak z-plane velocity magnitude occurs at an excitation voltage of Vrms $=17$ Volts.

\section{Conclusions}

This investigative research comprises three sections with the first two containing a study of the SJA in quiescent conditions. Therefore, some useful guidelines on the influence of clamping method and both geometrical and operating parameters on SJA design can be derived from this research. The results showed that the metal to metal clamping method introduced the best uniformity of the diaphragm motion as a result a higher displacement was generated. Moreover, it showed that the number of orifices and the space between them all influence the jet strength and velocity.

Furthermore, the results showed that it is desirable to operate the SJA at the Helmholtz frequency of the cavity instead of the diaphragm resonant frequency to avoid damaging the diaphragm material. It has been seen that use the Helmholtz resonance of the cavity and amplitude modulation excitation waveforms, the actuator exhibited significant blowing velocity, thus demonstrating that these excitation parameters have a useful function in practical engineering applications.

\section{Acknowledgment}

The first author, Itimad Azzawi, would like to express his gratitude to Iraqi organizations (HCED and University of Diyala) for their financial support during the PhD study. The authors would also like to thank the technicians at University of Leeds for their support.

\section{References}

[1] Ishtiaq A. C, Zhong S. Understanding the interaction of synthetic jet with the flat plate boundary layer. ICARME, Trivandrum, India. 2012 May.

[2] Monir HE, Tadjfar M, Bakhtian A. Tangential synthetic jets for separation control. Journal of Fluids and Structures. 2014 Feb 28; 45:50-65.

[3] Ishtiaq A. C, Zhong S. A single circular synthetic jet issued into turbulent boundary layer. Journal of Visualization. 2014 May $1 ; 17(2): 101-11$.

[4] Abed, Khuder N., and Itimad DJ Azzawi. "Control of Flow Separation Over Naca0015 Airfoil Using Synthetic Jet Actuators." Diyala Journal of Engineering Sciences 8, no. $4 \quad$ (2015): https://www.iasj.net/iasj?func=fulltext\&aId=109199

[5] Pack LG, Seifert A. Periodic excitation for jet vectoring and enhanced spreading. Journal of aircraft. 2001 May; 38(3):486-95. https://doi.org/10.2514/2.2788

[6] Smith BL, Glezer A. Jet vectoring using synthetic jets. Journal of Fluid Mechanics. 2002 May 10; 458:134. http://dx.doi.org/10.1017/S0022112001007406 
[7] Lim YL, Shamel MM. The Effect of Synthetic Jet Mixing on Enzyme Activity. Journal of Engineering Science and Technology. 2014; 85-95.

[8] Wang H, Menon S. Fuel-air mixing enhancement by synthetic microjets. AIAA journal. 2001 Dec; 39(12):2308-19.

[9] Jagannatha D, Chandratilleke T, Narayanaswamy R. Interactive Flow Behaviour and Heat Transfer Enhancement in a Microchannel with Cross-flow Synthetic Jet. International Journal of Emerging Multidisciplinary Fluid Sciences. 2010 Mar 1; 2(1):27-43.

[10] Trávníček Z, Tesař V. Annular synthetic jet used for impinging flow mass-transfer. International Journal of Heat and Mass Transfer. 2003 Aug 31; 46(17):3291-7.

[11] YAHYA, Samir Gh, Itimad DJ Azzawi, Mohammed k Abbas, and Ahmed AAG AL-RUBAIY. "Characteristics of Acoustic Drivers for Efficient Coupling to Thermoacoustic Machines." Proceedings of the International MultiConference of Engineers and Computer Scientists 2019 IMECS 2019, March 13-15, 2019, Hong Kong

[12] Yang AS. Design analysis of a piezoelectrically driven synthetic jet actuator. Smart Materials and Structures. 2009 Sep 23; 18(12):125004.

[13] Mane P, Mossi K, Rostami A, Bryant RG, Castro N. Piezoelectric actuators as synthetic jets: Cavity dimension effects. Journal of Intelligent Material Systems and Structures. 2007 May 30. http://jim.sagepub.com/cgi/content/abstract/18/11/1175

[14] Ugrina S. Experimental analysis and analytical modelling of synthetic jet cross flow interaction. PhD Thesis. Maryland: University of Maryland, USA, 2007.

[15] Mossi K, Mane P, Bryant R. Velocity profiles for synthetic jets using piezoelectric circular actuators. AIAA Paper. 2005 Apr 18; 2341:18-21. http://dx.doi.org/10.2514/6.2005-2341.

[16] Mane P. Experimental Design and Analysis of Piezoelectric Synthetic Jets in Quiescent Air. Master Dissertation. School of engineering Virginia Commonwealth University, 2005. http://scholarscompass.vcu.edu/etd/768/?show=full

[17] Myers OJ, Anjanappa M, Freidhoff C. Designing piezoelectric interdigitated microactuators using finite element analysis. Journal of Mechanical Design. 2010 Jun 1; 132(6):061004.

[18] Kim, Seon-Bae, Hyejin Park, Seung-Hyun Kim, H. Clyde Wikle, Jung-Hyun Park, and Dong-Joo Kim. "Comparison of MEMS PZT cantilevers based on $\mathrm{d} 31$ and $\mathrm{d} 33$ modes for vibration energy harvesting." Journal of microelectromechanical systems 22, no. 1 (2012): 26-33. 10.1109/JMEMS.2012.2213069

[19] Kang, Min-Gyu, Woo-Suk Jung, Chong-Yun Kang, and Seok-Jin Yoon. "Recent progress on PZT based piezoelectric energy harvesting technologies." In Actuators, vol. 5, no. 1, p. 5. Multidisciplinary Digital Publishing Institute, 2016. $\underline{10.3390 / \text { act5010005 }}$

[20] Mishra, Suvrajyoti, Lakshmi Unnikrishnan, Sanjay Kumar Nayak, and Smita Mohanty. "Advances in piezoelectric polymer composites for energy harvesting applications: a systematic review." Macromolecular Materials and Engineering 304, no. 1 (2019): 1800463. https://doi.org/10.1002/mame.201800463

[21] Knight, Ryan R., Changki Mo, and William W. Clark. "MEMS interdigitated electrode pattern optimization for a unimorph piezoelectric beam." Journal of electroceramics 26, no. 1-4 (2011): 14-22. https://doi.org/10.1007/s10832010-9621-8

[22] Watson, M. The Use of Synthetic Jet Actuators for the Enhanced Control of Separated Flows. PhD Thesis, University of Manchester, 2004. http://ethos.bl.uk/OrderDetails.do?uin=uk.bl.ethos.488291 
[23] Azzawi, Itimad DJ, X. Mao, and Artur J. Jaworski. "Design, fabrication and characterization of low speed open-jet wind tunnel." In Proceeings of World Congress on Engineering 2016, vol. 2, pp. 883-888. Newswood Limited, 2016. http://eprints.whiterose.ac.uk/99364/

[24] Jain M, Puranik B, Agrawal A. A numerical investigation of effects of cavity and orifice parameters on the characteristics of a synthetic jet flow. Sensors and Actuators A: Physical. 2011 Feb 28; 165(2):351-66. https://doi.org/10.1016/j.sna.2010.11.001

[25] Lv YW, Zhang JZ, Shan Y, Tan XM. Numerical investigation for effects of actuator parameters and excitation frequencies on synthetic jet fluidic characteristics. Sensors and Actuators A: Physical. 2014 Nov 1; 219:100-11. https://doi.org/10.1016/j.sna.2014.08.009

[26] Tang H, Zhong S. Modelling of the characteristics of synthetic jet actuators. AIAA Pap. 2005; 4748:2005. https://doi.org/10.2514/6.2005-4748

[27] Feero MA, Lavoie P, Sullivan PE. Influence of cavity shape on synthetic jet performance. Sensors and Actuators A: Physical. 2015 Mar 1; 223:1-0. https://doi.org/10.1016/j.sna.2014.12.004

[28] Milanovic I, Zaman KB. Synthetic Jets in Cross-Flow, Part II: Jets from Orifices of Different Geometry. AIAA Paper. 2002; 3715:23-6. https://doi.org/10.2514/6.2003-3715

[29] Jabbal, Mark, Stephen Liddle, Jonathan Potts, and William Crowther. "Development of design methodology for a synthetic jet actuator array for flow separation control applications." Proceedings of the Institution of Mechanical Engineers, Part G: Journal of Aerospace Engineering 227, no. 1 (2013): 110-124. https://doi.org/10.1177/0954410011428256

[30] The working principle of the scanning laser vibrometer is available at www.polytec.com if more detailed information is required.

[31] Razi, H. Circulation enhancement of synthetic jet actuators via multiple orifices. Msc Thesis, University of New South Wales, Sydney, Australia, 2012.

[32] Watson M, Jaworski AJ, Wood NJ. Contribution to the understanding of flow interactions between multiple synthetic jets. AIAA journal. 2003b Apr; 41(4):747-9. http://arc.aiaa.org/doi/abs/10.2514/2.2008.

[33] Blevins, R. and R. Plunkett. "Formulas for natural frequency and mode shape." (1986). 10.1115/1.3153712

[34]_Jeyalingam, Jonne. "Towards the noise reduction of synthetic jet actuators using lobed orifices." PhD diss., Brunel University London, 2018. http://bura.brunel.ac.uk/handle/2438/17118

[35] Tang H, Zhong S, Jabbal M, Garcillan L, Guo F, Wood N, Warsop C. Towards the design of synthetic-jet actuators for full-scale flight conditions. Flow, Turbulence and Combustion. 2007 Jun 1; 78(3-4):309-29.

[36] Hong, Guang. "Enabling micro synthetic jet actuators in boundary layer separation control using flow instability." In Proceedings of the 16th Australasian Fluid Mechanics Conference, 16AFMC. 2007. http://hdl.handle.net/10453/7236

[37] Tian, Ye, Louis Cattafesta, and Rajat Mittal. "Adaptive control of separated flow." In 44th AIAA aerospace Sciences meeting and Exhibit, p. 1401. 2006. https://doi.org/10.1063/1.2236305 


\section{Figure Captions List}

Figure 1 Schematic of SJA with piezo-ceramic diaphragm as active element.

Figure 2 Different ways of clamping: a) between two O-rings; b) using a neoprene rubber on one side; c) metal to metal clamps; d) assembled actuator unit.

Figure $3 \quad$ Polytec Scanning Vibrometer experimental setup.

Figure 4

Photograph of experimental setup (a) and block diagram of instrument layout (b).

Figure 5 Experimental set-up of the most efficient orifice spacing study.

Figure 6 The clamping method effect on the actuator performance at different clamping methods a) O-ring clamping b) metal to metal and neoprene rubber clamping.

Figure 7 The diaphragm displacement amplitude at different excitation frequencies at constant excitation voltage of $\mathrm{Vrms}=35 \mathrm{~V}$.

Figure 8 Time history of the $\mathrm{x}$-plane velocity magnitude measured at $\mathrm{H} / \mathrm{D}_{\mathrm{c}}=0.093$ and $\mathrm{Y} / \mathrm{D}_{\mathrm{o}}=0.5$ above the orifice centerline $(\mathrm{Vrms}=35$ volts, excitation frequency $=1800 \mathrm{~Hz}$ ).

Figure 9 Time history of the velocity magnitude measured in the stream-wise at $H / \mathrm{D}_{\mathrm{c}}=0.093$; $\mathrm{Y} / \mathrm{D}_{\mathrm{o}}=0.5 ; \mathrm{Y} / \mathrm{D}_{\mathrm{o}}=4$ and $\mathrm{Y} / \mathrm{D}_{\mathrm{o}}=6$ above the orifice centreline $(\mathrm{Vrms}=35$ volt and excitation frequency $=1800 \mathrm{~Hz}$ ).

Figure $10 \quad$ Power spectral density of z-plane velocity magnitude at $H / \mathrm{D}_{\mathrm{c}}=0.093 ; \mathrm{Y} / \mathrm{D}_{\mathrm{o}}=0.5$ and $\mathrm{Y} / \mathrm{D}_{\mathrm{o}}=15$ above the orifice centreline $(\mathrm{Vrms}=35$ volt and excitation frequency $=1800$ $\mathrm{Hz}$ ).

Figure 11 Experimental data of the frequency response of the synthetic jet actuator at different numbers of orifices (Vrms $=35$ volts, metal-to-metal clamping).

Figure 12 Calculated Helmholtz frequency against cavity height for three orifices diameters.

Figure $13 \mathrm{x}$-plane velocity magnitude profile $1 \mathrm{~mm}$ above the orifice exit plane for synthetic jet actuators with $\mathrm{D}_{\mathrm{o}}=1.2 \mathrm{~mm}$ but different cavity height $(f=1000 \mathrm{~Hz}$, Vrms $=35$ volt $)$.

Figure $14 \quad$ Final geometrical layout of the single SJA.

Figure $15 \quad$ An example of PSD in a wake area airfoil trailing edge [37].

Figure 16 The creation of a modulated signal by adding a carrier with a modulated wave. 
Figure 17 Power spectral density at $\mathrm{Y} / \mathrm{D}_{\mathrm{o}}=0.5$, a) Un-modulated jets $\left(f \mathrm{c}=f_{\mathrm{H}}=1000 \mathrm{~Hz}\right)$, b) Modulated jets $\left.\left(f \mathrm{c}=f_{\mathrm{H}}=1000 \mathrm{~Hz}, f_{\mathrm{m}}=75 \mathrm{~Hz}\right), \mathrm{c}\right)$ Modulated jets $\left(f_{\mathrm{c}}=f_{\mathrm{H}}=1000 \mathrm{~Hz}, f_{\mathrm{m}}=\right.$ $150 \mathrm{~Hz})$.

Figure 18 Synthetic jet velocity at Vrms = 35 volts for a) Un-modulated synthetic jets, b) Modulated wave $\left.\left(f \mathrm{c}=1000 \mathrm{~Hz}, f_{\mathrm{m}}=75 \mathrm{~Hz}\right), \mathrm{c}\right)$ Modulated $(f \mathrm{c}=1000 \mathrm{~Hz}, \mathrm{fm}=150 \mathrm{~Hz})$.

Figure 19 Peak jet velocity for both modulated and un-modulated wave at $\left(\mathrm{Y} / \mathrm{D}_{\mathrm{o}}=0.5, f \mathrm{c}=f_{\mathrm{H}}\right.$ $=1000 \mathrm{~Hz})$.

Figure 20

Experimental data of the frequency response of the synthetic jet actuator at different numbers of orifices (Vrms $=35$ volts, metal-to-metal clamping).

\section{Table Caption List}

Table 1 Geometrical properties of the baseline synthetic jet actuator. 


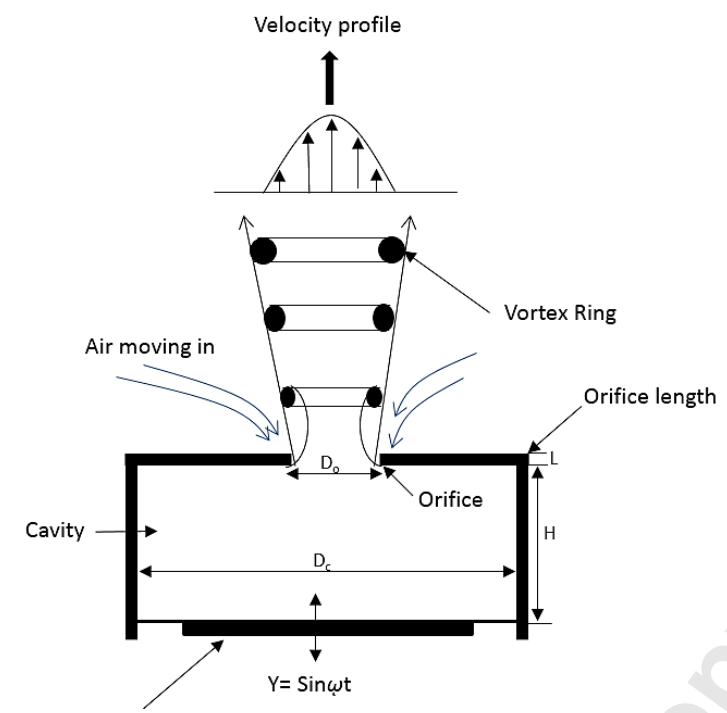

Piezoceramic/oscillatory diaphragm

Figure 1: Schematic of SJA with piezo-ceramic diaphragm as active element. 


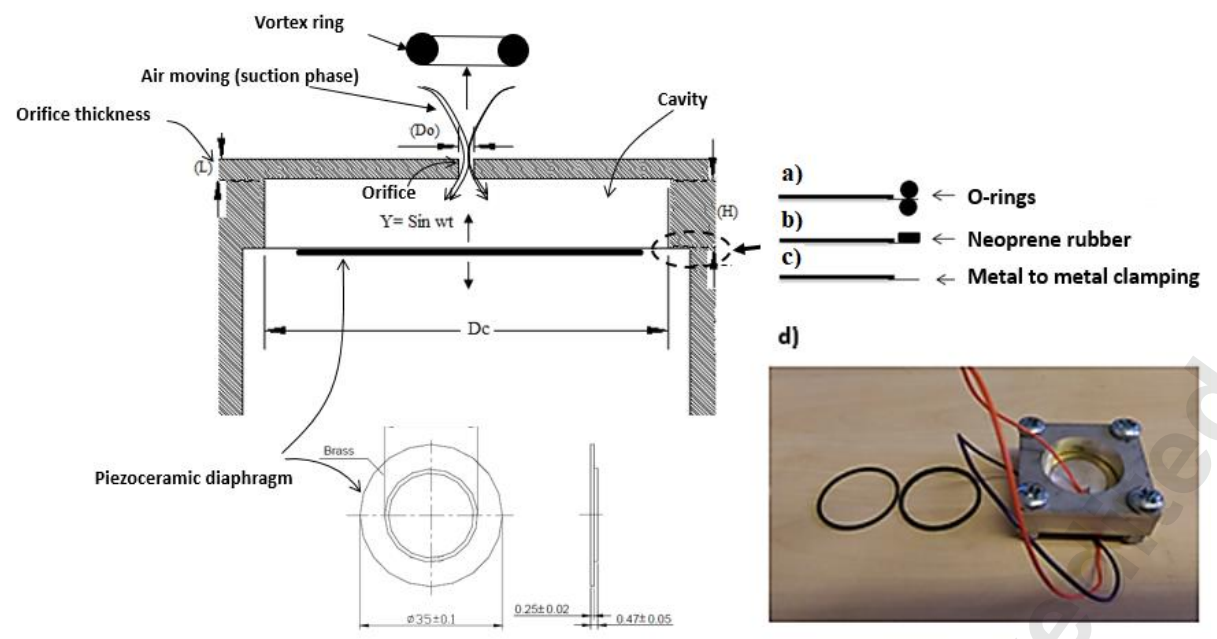

Figure 2: Different ways of clamping: a) between two O-rings; b) using a neoprene rubber on one side; c) metal to metal clamps; d) assembled actuator unit. 


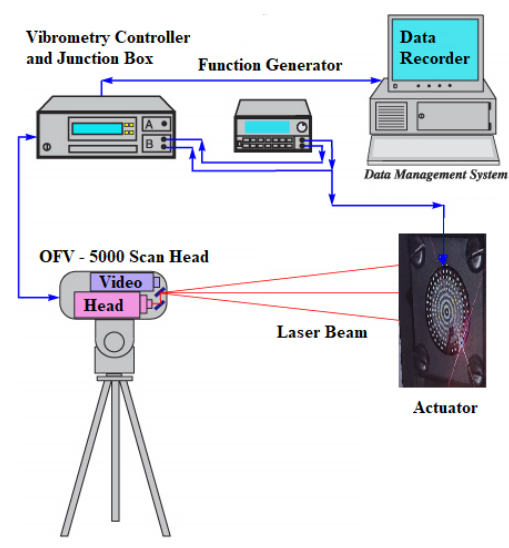

Figure 3: Polytec Scanning Vibrometer experimental setup. 


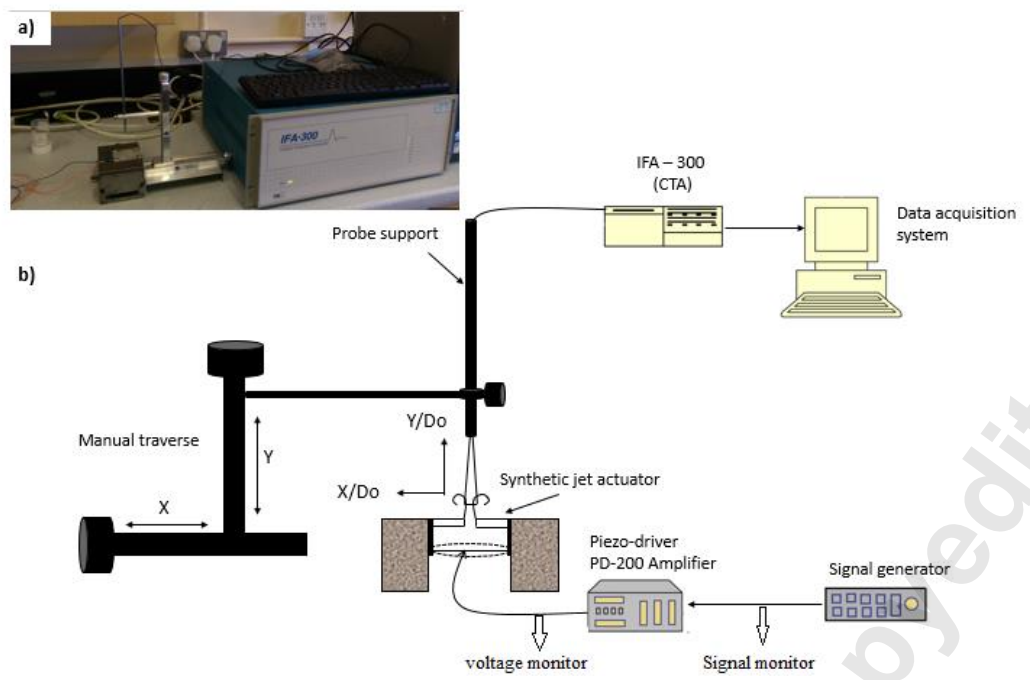

Figure 4: Photograph of experimental setup (a) and block diagram of instrument layout (b). 
Journal of Fluids Engineering. Received June 15, 2020;

Accepted manuscript posted October 28, 2020. doi:10.1115/1.4048930

Copyright (c) 2020 by ASME

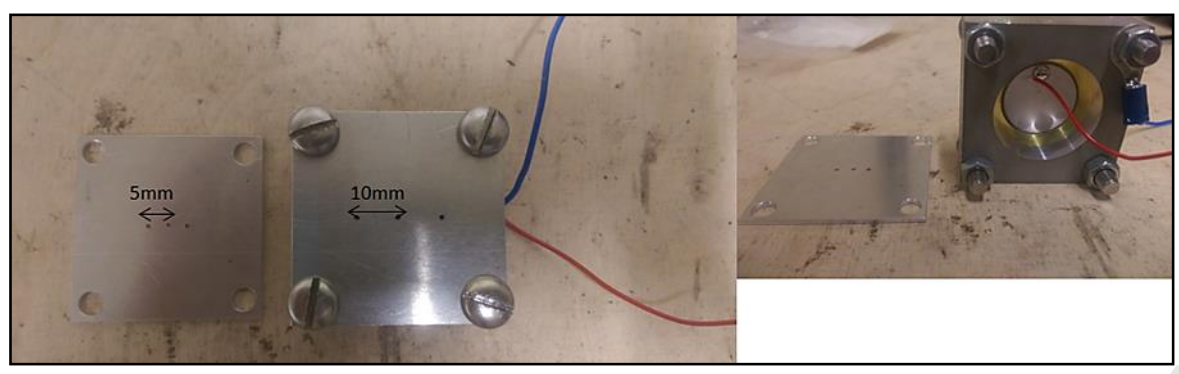

Figure 5: Experimental set-up of the most efficient orifice spacing study. 

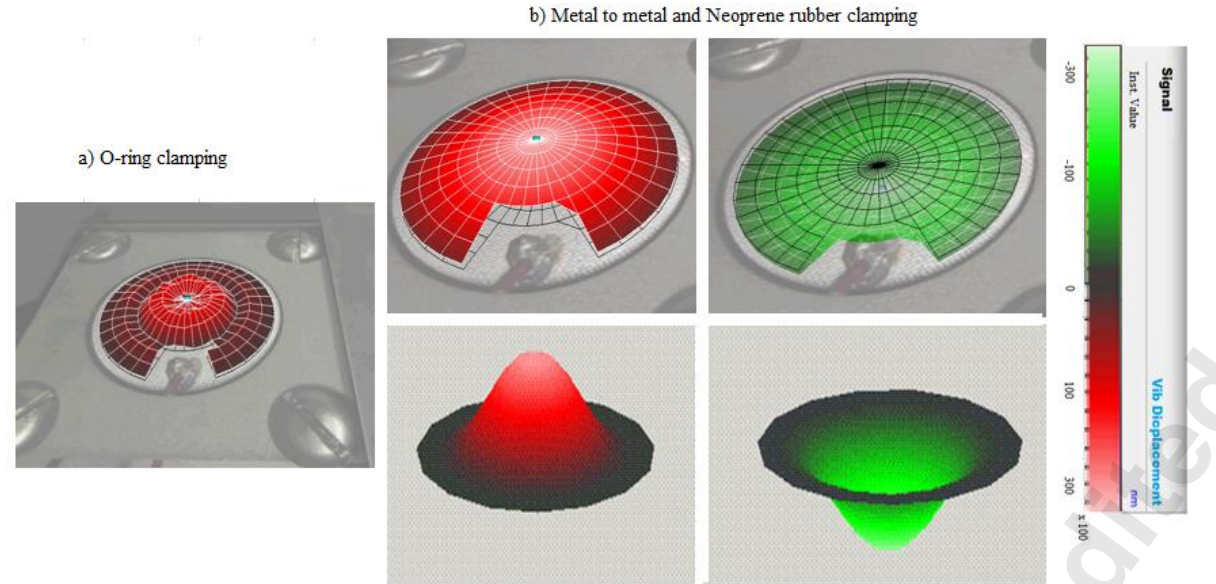

Figure 6: The clamping method effect on the actuator performance at different clamping methods a) O-ring clamping b) metal to metal and neoprene rubber clamping. 


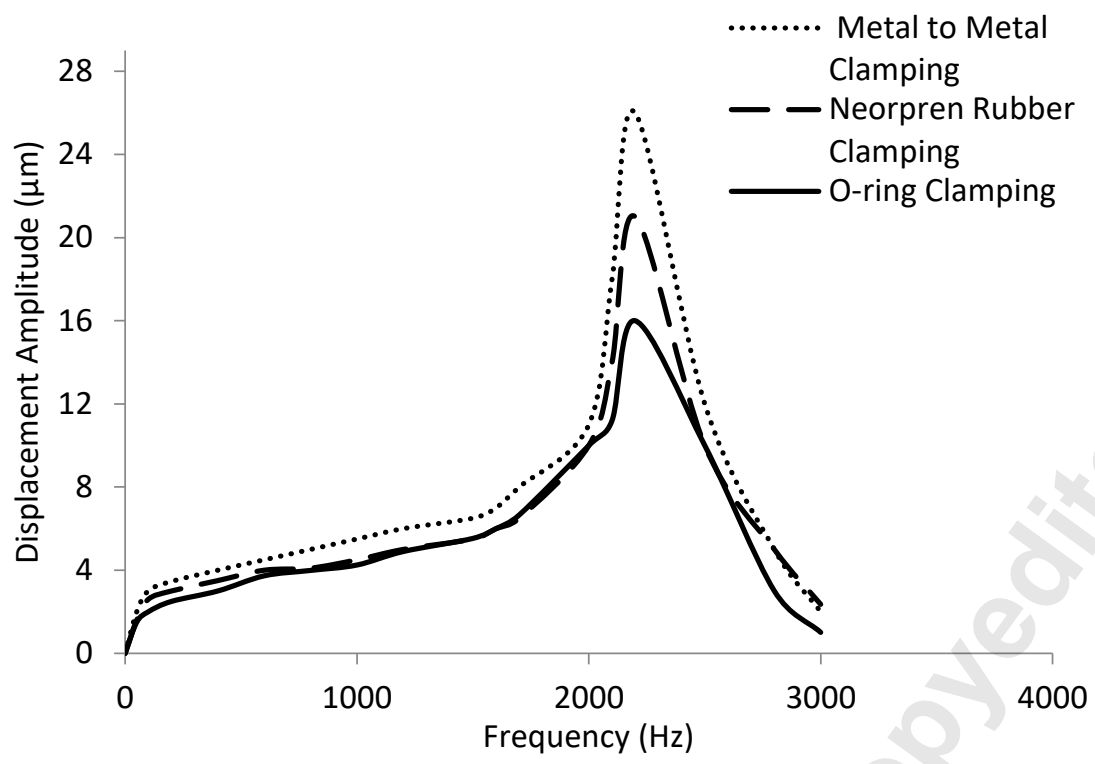

Figure 7: The diaphragm displacement amplitude at different excitation frequencies at constant excitation voltage of Vrms = $35 \mathrm{~V}$. 


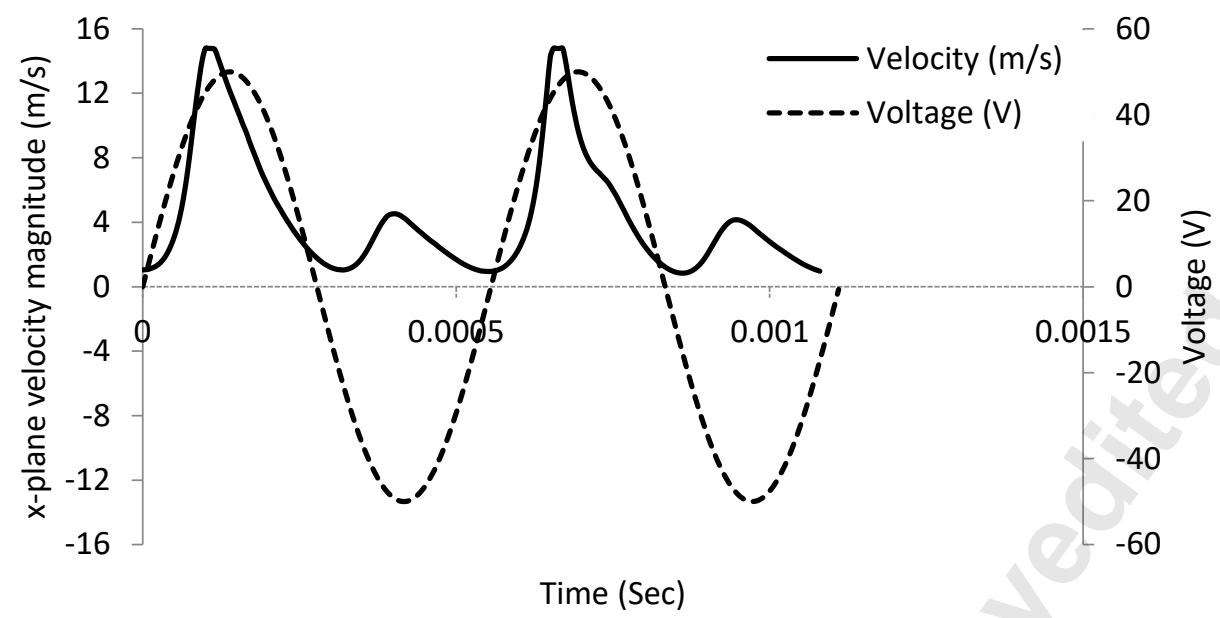

Figure 8: Time history of the $\mathrm{x}$-plane velocity magnitude measured at $\mathrm{H} / \mathrm{D}_{\mathrm{c}}=0.093$ and $\mathrm{Y} / \mathrm{D}_{\mathrm{o}}=0.5$ above the orifice centerline $($ Vrms $=35$ volts, excitation frequency $=1800 \mathrm{~Hz})$. 


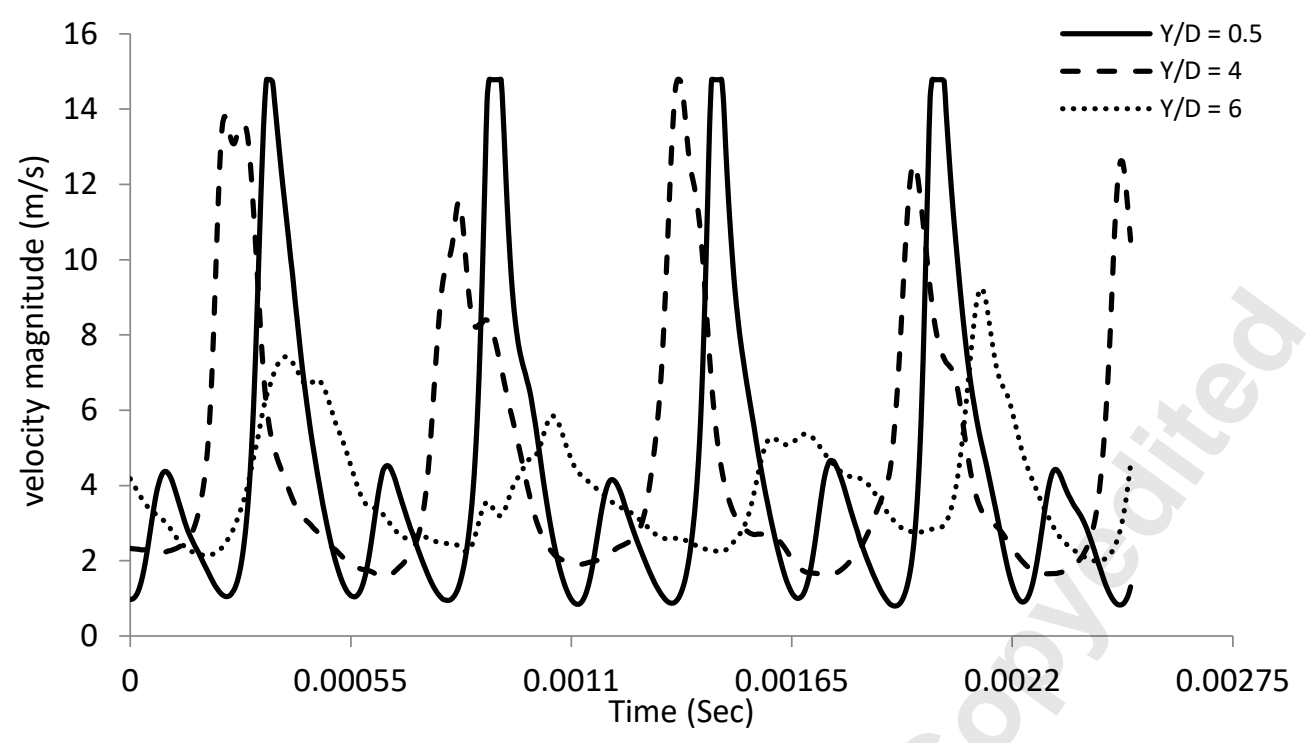

Figure 9: Time history of the velocity magnitude measured in the stream-wise at $H / \mathrm{D}_{\mathrm{c}}=0.093 ; \mathrm{Y} / \mathrm{D}_{\mathrm{o}}=0.5 ; \mathrm{Y} / \mathrm{D}_{\mathrm{o}}=4$ and $\mathrm{Y} / \mathrm{D}_{\mathrm{o}}=6$ above the orifice centreline $(\mathrm{Vrms}=35$ volt and excitation frequency $=1800 \mathrm{~Hz})$. 


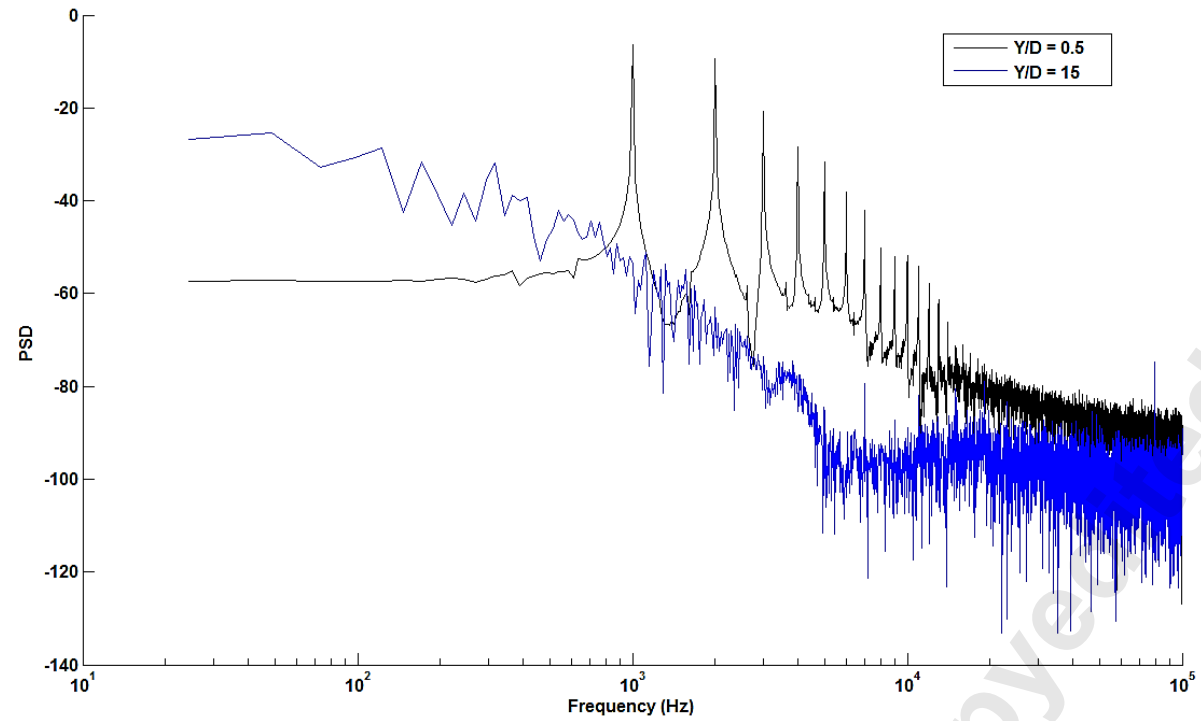

Figure 10: Power spectral density of z-plane velocity magnitude at $\mathrm{H} / \mathrm{D}_{\mathrm{c}}=0.093 ; \mathrm{Y} / \mathrm{D}_{\mathrm{o}}=0.5$ and $\mathrm{Y} / \mathrm{D}_{\mathrm{o}}=15$ above the orifice centreline $($ Vrms $=35$ volt and excitation frequency $=1800 \mathrm{~Hz})$. 

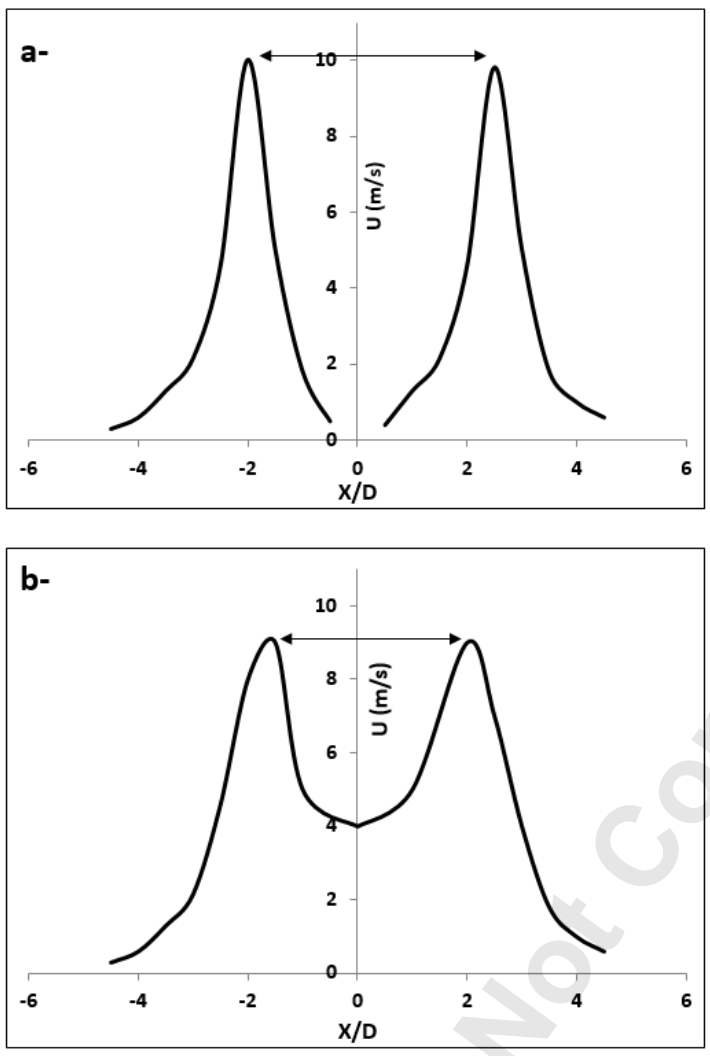

Figure 11: Radial velocity profile in the cross-stream direction $\left(\mathrm{X} / \mathrm{D}_{\mathrm{o}}\right)$ at location of $\mathrm{Y} / \mathrm{D}_{\mathrm{o}}=3$ for orifice spaced a) $10 \mathrm{~mm}$ and b) $5 \mathrm{~mm}(f=1000 \mathrm{~Hz}, \mathrm{~V}=35 \mathrm{Vrms})$. 


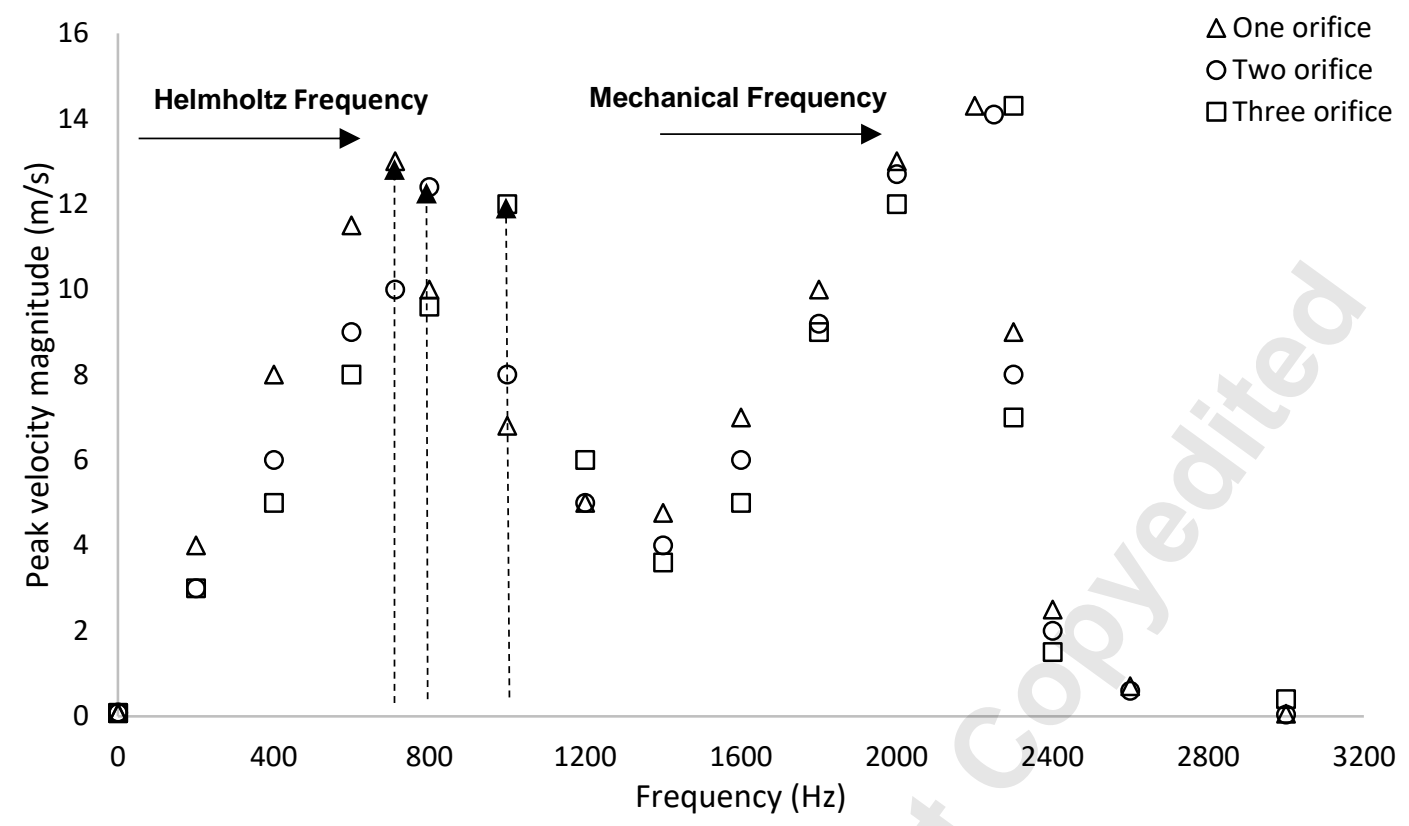

Figure 12: Experimental data of the frequency response of the synthetic jet actuator at different numbers of orifices $($ Vrms $=$ 35 volts, metal-to-metal clamping). 


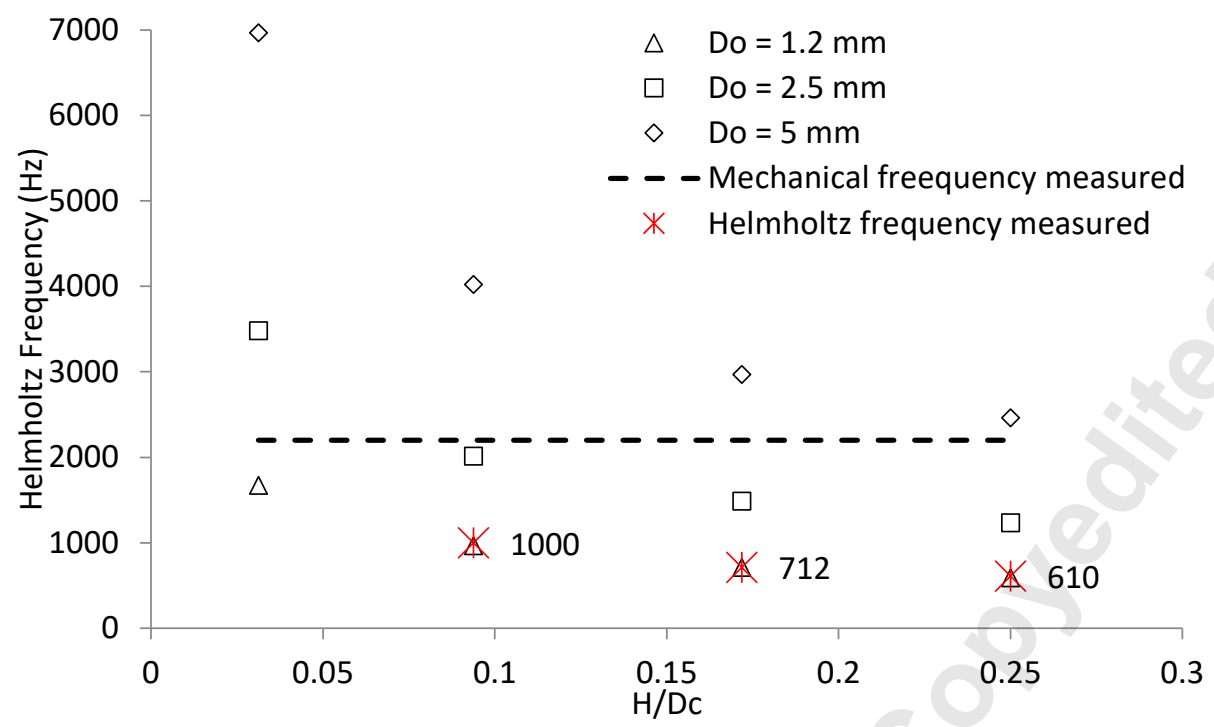

Figure 13: Calculated Helmholtz frequency against cavity height for three orifices diameters. 


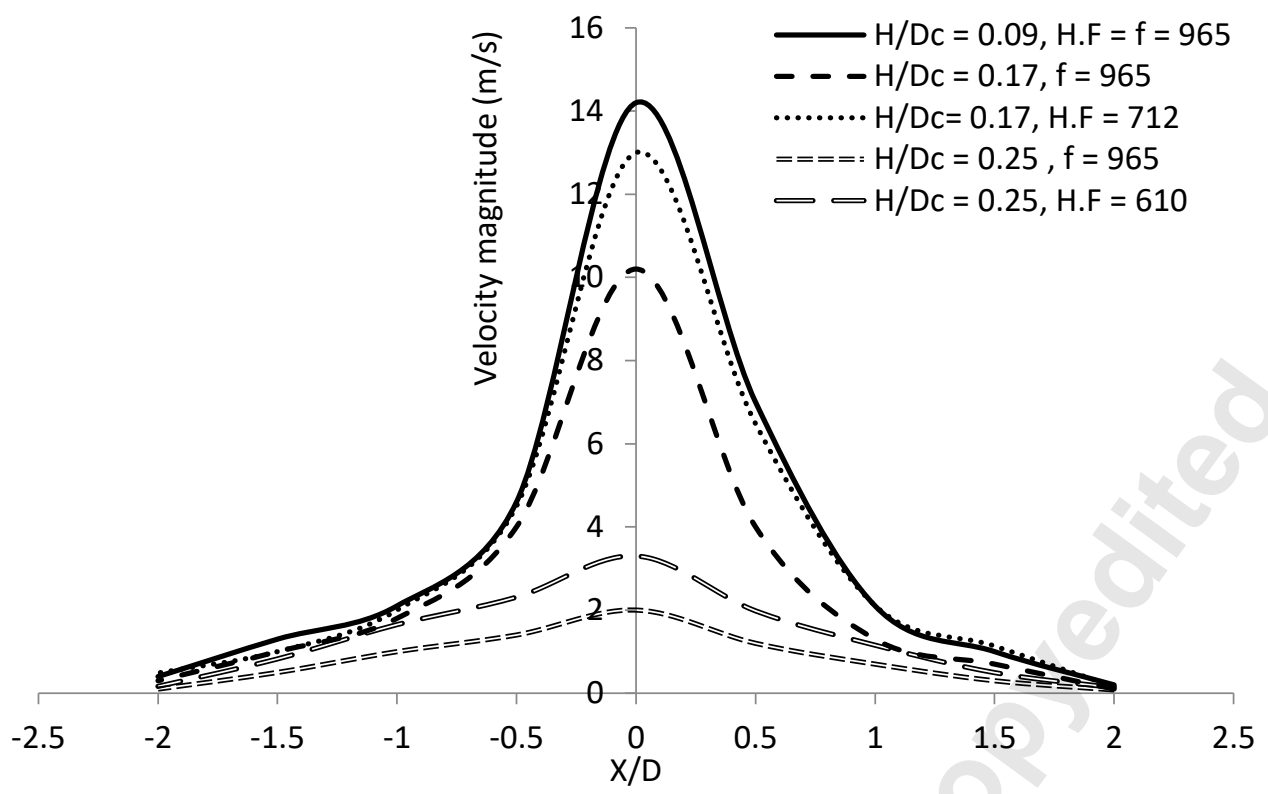

Figure 14: x-plane velocity magnitude profile $1 \mathrm{~mm}$ above the orifice exit plane for synthetic jet actuators with $\mathrm{D}_{\mathrm{o}}=1.2 \mathrm{~mm}$ but different cavity height $(f=1000 \mathrm{~Hz}, \mathrm{Vrms}=35$ volt $)$. 


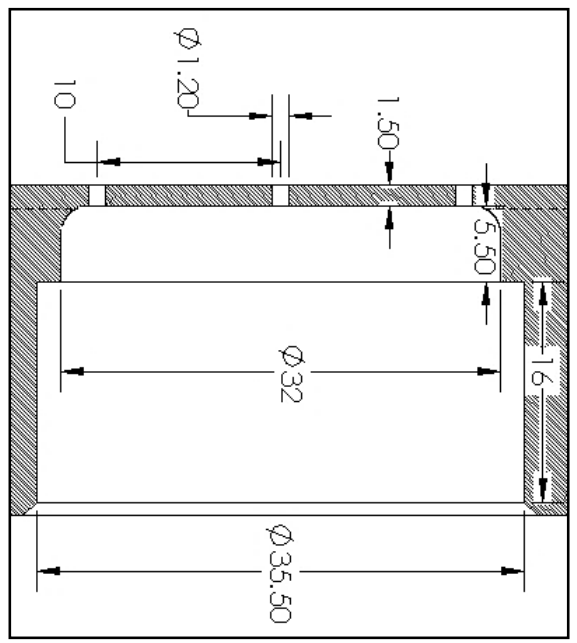

Figure 15: Final geometrical layout of the single SJA. 


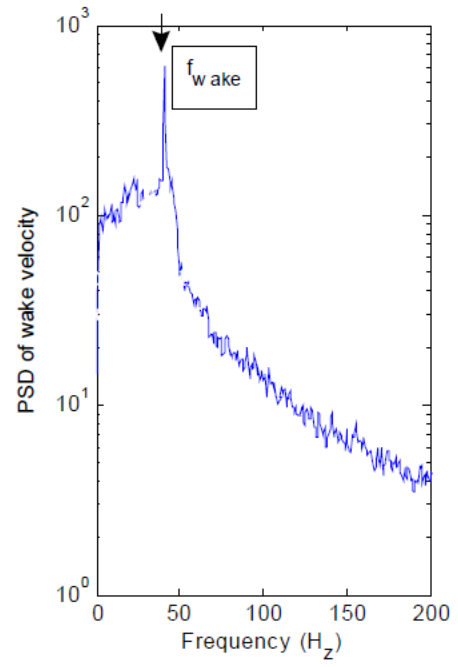

Figure 16: An example of PSD in a wake area airfoil trailing edge [37]. 


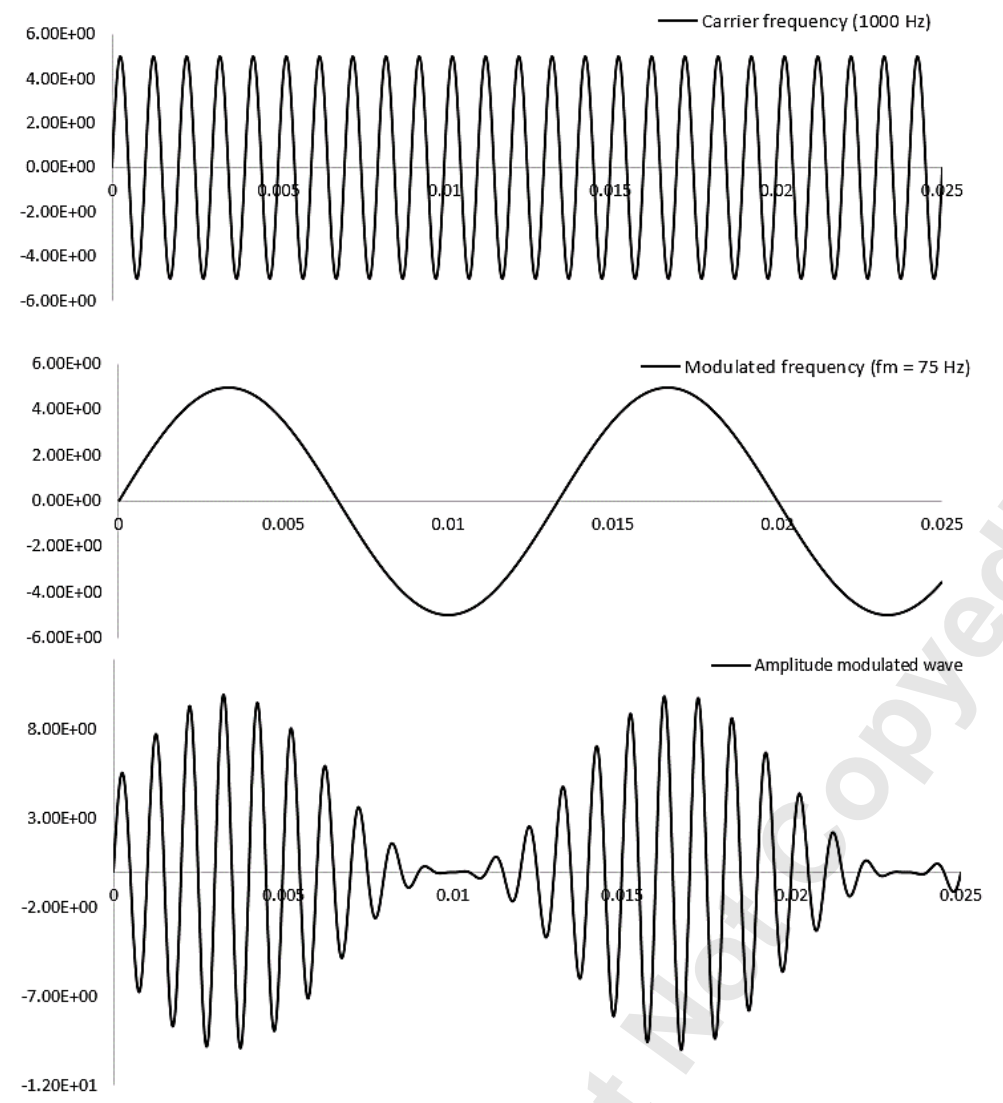

Figure 17: The creation of a modulated signal by adding a carrier with a modulated wave. 

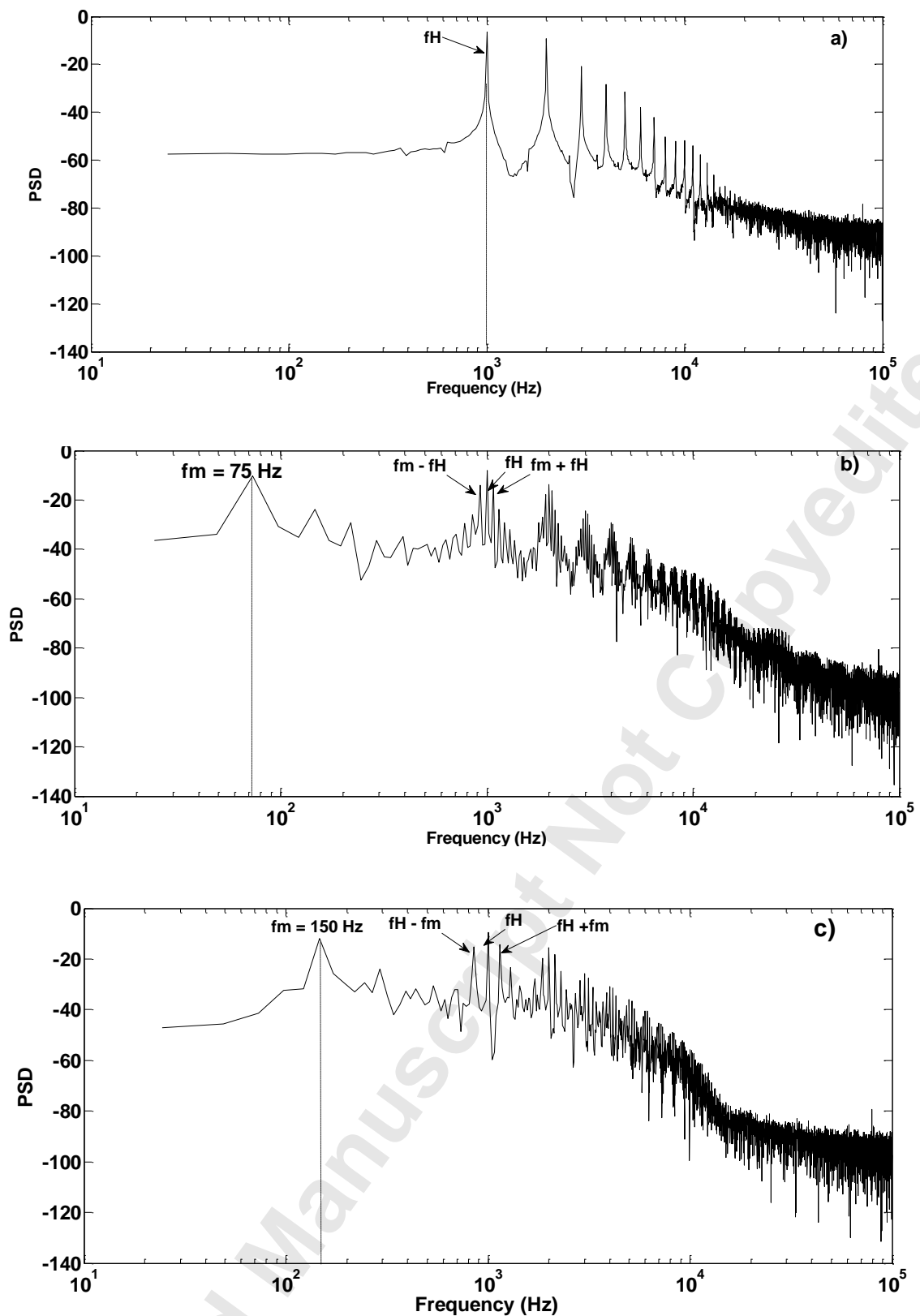

Figure 18: Power spectral density at $\mathrm{Y} / \mathrm{D}_{\mathrm{o}}=0.5$, a) Un-modulated jets $\left.\left(f_{\mathrm{c}}=f_{\mathrm{H}}=1000 \mathrm{~Hz}\right), \mathrm{b}\right)$ Modulated jets $\left(f \mathrm{c}=f_{\mathrm{H}}=1000\right.$ $\left.\left.\mathrm{Hz}, f_{\mathrm{m}}=75 \mathrm{~Hz}\right), \mathrm{c}\right)$ Modulated jets $\left(f_{\mathrm{c}}=f_{\mathrm{H}}=1000 \mathrm{~Hz}, f_{\mathrm{m}}=150 \mathrm{~Hz}\right)$. 

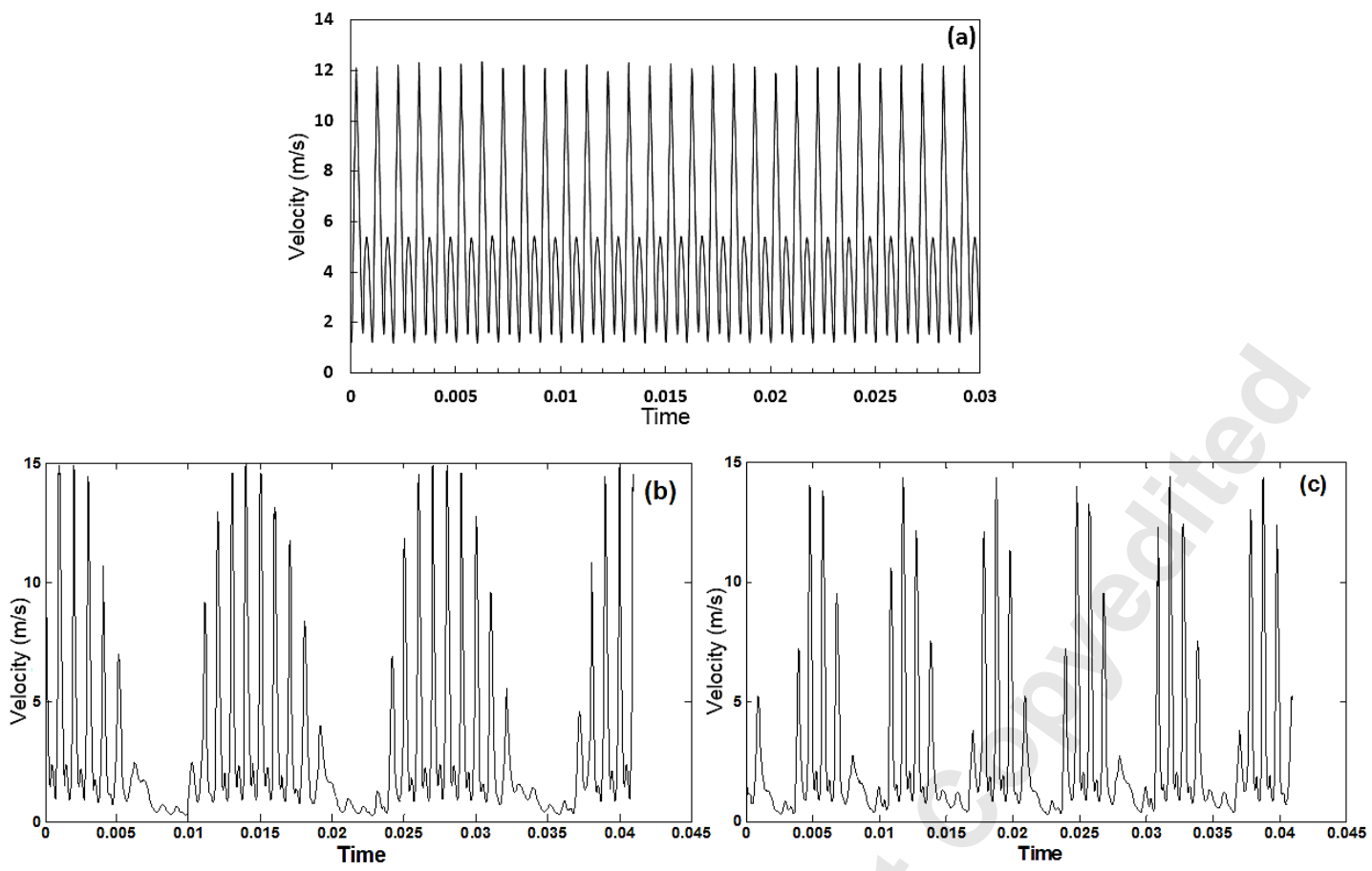

Figure 19: Synthetic jet velocity at Vrms $=35$ volts for a) Un-modulated synthetic jets, b) Modulated wave $\left(f \mathrm{c}=1000 \mathrm{~Hz}, f_{\mathrm{m}}\right.$ $=75 \mathrm{~Hz}), \mathrm{c})$ Modulated $(\mathrm{fc}=1000 \mathrm{~Hz}, \mathrm{fm}=150 \mathrm{~Hz})$. 


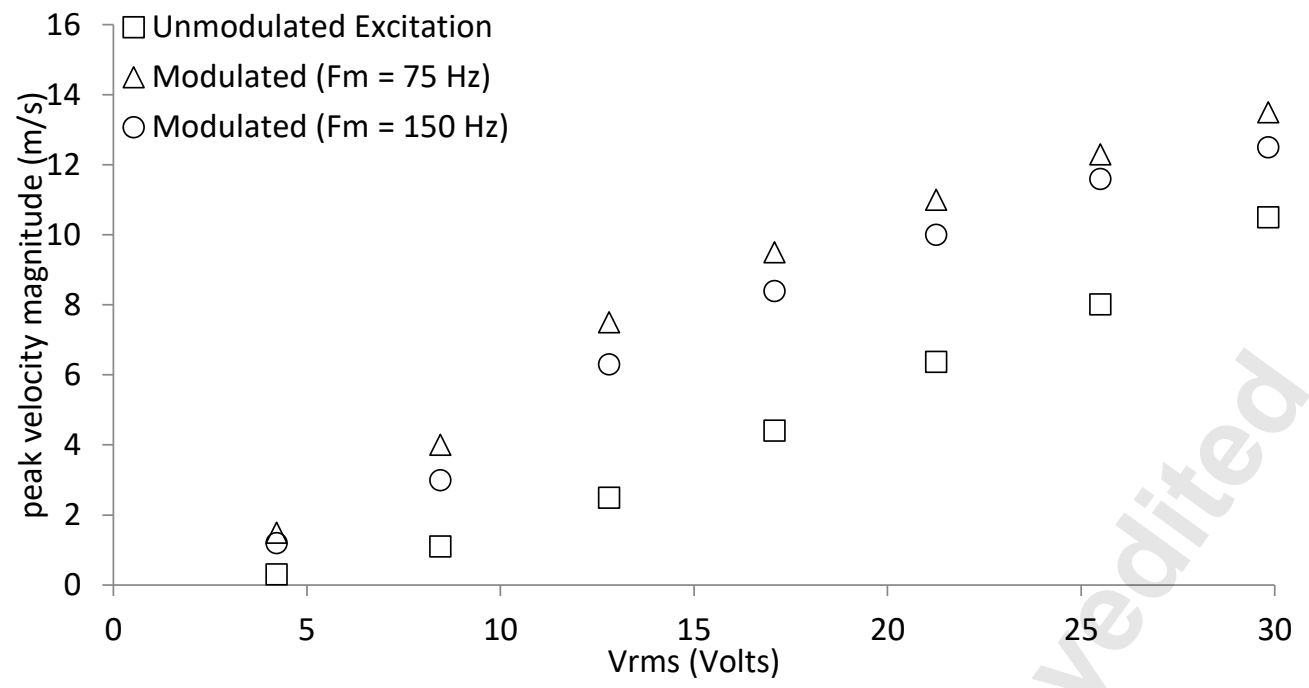

Figure 20: Peak jet velocity for both modulated and un-modulated wave at $\left(\mathrm{Y} / \mathrm{D}_{\mathrm{o}}=0.5, f \mathrm{c}=f_{\mathrm{H}}=1000 \mathrm{~Hz}\right)$. 
Table 1: Geometrical properties of the baseline synthetic jet actuator.

\begin{tabular}{|c|c|}
\hline Actuator Parameters & Value \\
\hline Orifice diameter $\left(\mathrm{D}_{\mathrm{o}}\right)$ & $1.2 \mathrm{~mm}$ \\
\hline Orifice Length $(\mathrm{L})$ & $1.5 \mathrm{~mm}$ \\
\hline Cavity Height $(\mathrm{H})$ & $3 \mathrm{~mm}$ \\
\hline Cavity Diameter $\left(\mathrm{D}_{\mathrm{c}}\right)$ & $32 \mathrm{~mm}$ \\
\hline Piezo-ceramic Diaphragm & PC FT-27T-3.9A1 \\
\hline
\end{tabular}

